\title{
Die gegenwärtige Entwicklung des Rundfunks in der Bundesrepublik Deutschland und die katholische Kirche
}

\author{
Plädoyer für eine Standortüberprüfung \\ von Hans Wolfgang Rombach
}

I.

Unsere Zeit ist einer gewissen Art von Worten offenbar besonders zugetan, deren wesentlichste Eigenschaften ihre eingängige Konturenlosigkeit und ihre erstaunliche Fähigkeit sind, Wünsche und Abneigungen, Zustimmungen und Verweigerungen wie ein Schwamm in sich aufzusaugen. Ein typisches Beispiel dafür ist das gegenwärtig in der öffentlichen Diskussion augenscheinlich so hoch favorisierte Wort "Medien «, vor allem, wenn es, versehen mit dem erwartungsgesättigten Zusatz »neue « auftritt. Jeder, der etwas auf sich hält, vor allem jeder, der in dem Bereich mitreden will, der früher schlicht mit »Rundfunk « bezeichnet wurde, hat diese Modevokabel längst selbstverständlich, ja meist unreflektiert in seinen Wortschatz übernommen; dabei könnte ihn ein kurzer Blick in Duden's Fremdwörterbuch schnell belehren, daß "Medium" nichts anderes als »Mittel, Mittelglied, vermittelndes Element « heißt und damit ein recht ungenauer, fast immer präzisierender Erläuterung bedürftiger Begriff ist.

Zumindest dort, wo die Diskussion um den Rundfunk kreist, empfiehlt es sich daher, auf dieses neumodische Accessoire der deutschen Sprache zu verzichten, weil man anderenfalls ohne den Ariadnefaden ständiger Definitionen kaum noch aus dem Labyrinth der meist kunstvoll verflochtenen Argumente und Gegenargumente herausfindet. So soll in den nachfolgenden Darlegungen - wo immer möglich - nicht von Medien, sondern stets von Rundfunk gesprochen werden.

\section{II.}

Dieser Rundfunk befindet sich in einer gewichtigen, in allen ihren Konsequenzen noch kaum überschaubaren Phase der Veränderung; sie kann ihrer Bedeutung nach durchaus mit dem Einbruch des Fernsehens in die deutsche Rundfunkwelt vor mehr als drei Jahrzehnten verglichen werden.

Ursache dieser Veränderung sind bedeutende technische Neuerungen, die es möglich machen, die bislang begrenzte Zahl terrestrischer Frequenzen für die Übertragung von Rundfunkprogrammen und ihre dadurch erzwungene Begrenzung zu überwinden. Wenn dieser Prozeß auch noch keineswegs abgeschlossen ist, so ist er doch heute schon - etwa gemessen am Zustand vor einem Jahrzehnt - beträchtlich fortgeschritten und entwickelt sich mit der Eigendynamik technischer Innovationen. In absehbarer Zeit wird es daher nicht nur möglich sein, der weitaus überwiegenden Zahl der deutschen Rundfunkteilnehmer einen qualitativ wesentlich verbesserten Hörfunk-

Hans Wolfgang Rombach, Ministerialdirigent a. D., bis 1984 Leiter der Abteilung Recht und Verwaltung in der Staatskanzlei des Landes Nordrhein-Westfalen und damit zuständig für Rundfunkrecht. Der Autor war bis 1984 zweiter Vertreter der Landesregierung beim WDR und ist seit 1985 Mitglied des Rundfunkausschusses nach dem Landesgesetz NW über die vorläufige Weiterverbreitung von Rundfunkprogrammen in Kabelanlagen. 
und Fernsehempfang anzubieten; - auch die Zahl der Rundfunkprogramme wird erheblich vermehrbar sein. Damit wird zugleich, zumindest aus der Sicht der Frequenzknappheit, die rechtliche Legitimation entfallen, Rundfunk in der Bundesrepublik wie bisher nur durch die öffentlich-rechtlichen Rundfunkanstalten ARD und ZDF betreiben zu lassen, deren organisatorische Struktur dem Grundsatz des »Binnenpluralismus «, d.h. der Teilhabe der gesellschaftlich relevanten Kräfte am Rundfunk durch Mitgliedschaft in den Organen der Rundfunkanstalten und gruppenspezifische Einwirkung auf deren Entscheidungen folgt. Neben diesen bisher allein praktizierten und daher dem deutschen Rundfunkteilnehmer vertrauten Typ von Rundfunk treten jetzt neue Rundfunkveranstalter. Sie organisieren sich auf privat-rechtlicher Grundlage und wollen insgesamt ein System von Rundfunk konstituieren, bei dem im Wege der »Außenpluralität« Meinungsvielfalt hergestellt wird, indem jeder dieser Veranstalter seinen - je eigenen - Beitrag zu einem Gesamtspektrum von Meinungsvielfalt leistet. Sicher ist der privatrechtlich verfaßte Rundfunk derzeit noch relativ weit von diesem Ziel entfernt; nicht zu leugnen ist jedoch, daß er sich auf dieses Ziel hin zu entwickeln beginnt, und daß diese Entwicklung sicherlich nicht mehr als ein etwas dubioses Experiment mit ungewissen Erfolgschancen angesehen werden kann.

III.

Daß diese Entwicklung - trotz der in bezug auf sie in der Bundesrepublik schon seit Jahren mit Härte, ja Verbissenheit geführten Auseinandersetzung der Fachleute und Politiker - von der Bevölkerung kaum zur Kenntnis genommen wird, folgt aus der schlichten Tatsache, daß, gemessen an der Gesamtzahl der Rundfunkteilnehmer, bisher nur recht wenige von ihnen die Programme der neuen privaten Rundfunkveranstalter empfangen, sich einen persönlichen Eindruck von ihnen verschaffen, Altes und Neues kritisch gegeneinander abwägen und so zu einer von der Diskussion der Fachleute nicht vorgeprägten eigenen Meinung kommen können. Diese Programme sind nämlich über terrestrische Frequenzen nicht - bzw. noch nicht - empfangbar; sie werden vielmehr von Satelliten abgestrahlt und über Erdstationen der Bundespost in Kabelanlagen eingespeist, so daß nur derjenige diese Programme empfangen kann, der an eine solche Kabelanlage angeschlossen ist. Die Verkabelung der Bundesrepublik schreitet aber, entgegen ersten, höchst optimistischen Prognosen der dafür zuständigen Bundespost, auch jetzt noch verhältnismäßig langsam fort, war zudem auch jahrelang durch eine schwerfällige Diskussion über die technische Leistungsfähigkeit verschiedener Kabelarten (Kupferkoaxial- oder Glasfaserkabel) belastet, und wurde in einzelnen Ländern, je nach deren rundfunkpolitischem Standort, auch durch mancherlei direkte oder indirekte Maßnahmen oder Einflußnahmen behindert bzw. verzögert. All dies - die rundfunkpolitische Dauerdiskussion, der Verkabelungsstreit, die Unsicherheit über die für den einzelnen Rundfunkteilnehmer bei einem Kabelanschluß entstehenden Kosten und manches mehr - hat zu einer gewissen allgemeinen Verunsicherung geführt, deren Folge sich auch jetzt noch in deutlicher Zurückhaltung gegenüber den Programmangeboten der neuen Rundfunkveranstalter selbst dort ausdrückt, wo die technischen Möglichkeiten ihren Empfang zulassen.

Das beträchtliche Mißverhältnis zwischen der Empfangbarkeit der Programme der öffentlich-rechtlichen und privat-rechtlichen Rundfunkveranstalter hat die Bundesregierung im Juni 1986 im Rahmen ihres "Programms zur Verbesserung der Rahmenbedingungen für den privaten Rundfunk « wie folgt dargestellt: Die Veranstaltungen des öffentlich-rechtlichen Rundfunks können derzeit von ca. 25 Mio Haushalten empfan- 
gen werden, diejenigen des privat-rechtlichen Rundfunks von ca. 1 Mio Haushalten. ARD und ZDF verfügen über dichte, mit wenigen Ausnahmen - etwa in topographisch schwierigen Gegenden - flächendeckende terrestrische Sendernetze; die privaten Rundfunkveranstalter sind bislang allein auf die Übertragung ihrer Programme via Satellit und Kabelnetz angewiesen, wobei auch hier vorerst nur begrenzte Kapazitäten bereitstehen. Zudem haben ARD und ZDF sich auch in diesem Übertragungsbereich bereits - teilweise gemeinsam mit ausländischen öffentlich-rechtlichen oder staatlichen Programmveranstaltern - Übertragungsmöglichkeiten gesichert, das ZDF bei »3sat «, die ARD bei »1 plus «. Diese Nutzungsrechte beruhen in einigen Ländern auf politischen Willensentscheidungen der jeweiligen Regierungen, in anderen auf rundfunkrechtlichen Regelungen, welche den öffentlich-rechtlichen Rundfunkanstalten - auch im technischen Bereich - die gleichen Möglichkeiten zusichern wie den privaten Rundfunkveranstaltern: rundfunkpolitisch ist dies Ausfluß einer staatlichen Bestands- und Entwicklungsgarantie « für die öffentlich-rechtlichen Rundfunkveranstalter.

ARD und ZDF erhalten zudem regelmäßige, gesicherte Einkünfte in Höhe von derzeit über 6 Mio DM, zum weitaus größten Teil aus einer öffentlich-rechtlichen Gebühr, die in den letzten zwei Jahrzehnten wiederholt angehoben worden ist, zum kleineren Teil aus Erträgen der Rundfunkwerbung. Demgegenüber sind die privatrechtlichen Rundfunkveranstalter zur Finanzierung ihrer Programme ausschließlich auf Einnahmen aus Rundfunkwerbung verwiesen, soweit ihre Rechtsträger nicht Gewinne aus anderen Unternehmensbereichen in die Finanzierung privater Rundfunkprogramme investieren.

In deutlichem Gegensatz zu der zumindest abwartenden Haltung vieler Rundfunkteilnehmer gegenüber dem privat-rechtlich verfaßten Rundfunk wird in der deutschen Öffentlichkeit die rundfunk-politische Auseinandersetzung über diese Frage nun schon seit Jahren zwischen den - wirklichen oder vermeintlichen - Fachleuten aller Art, den "Insidern " und denen, die sich dafür halten, den öffentlich-rechtlichen Rundfunkanstalten und ihren privat-rechtlichen Kontrahenten, zwischen Rundfunk und Presse, vor allem aber zwischen den politischen Parteien mit einem gewaltigen Aufwand an Argumenten, Energie und Heftigkeit, oft sogar mit einer Verbissenheit geführt, die sich mehr als einmal der Qualität rundfunk-politischer Glaubenskriege näherte. Einzelheiten dieses Streites sollen hier nicht dargestellt werden; denn dies wäre nur in sehr verkürzter Form möglich. Jede verkürzte Darstellung aber würde mit Sicherheit sogleich dem Vorwurf unzulässiger Vereinfachung, subjektiver Ein- oder Ausgrenzung von einem der Kontrahenten als fundamental angesehener Thesen oder Gegenthesen oder gar dem Vorwurf der Klitterei begegnen. Große Mengen einschlägiger Publikationen, Niederschriften über Parlamentsdebatten, Gerichtsentscheidungen, Berichte über Medienkongresse und Akademietagungen und vieles mehr füllen mittlerweile Regale von Bibliotheken und stehen dort jedermann zur Unterrichtung zur Verfügung. Vieles von alledem wird überdies bis heute in regelmäßigen Abständen über Presse und Rundfunk mit gelegentlich ermüdender Monotonie wiederholt, und beide Seiten hören dabei nicht auf, die alleinige Richtigkeit ihrer Thesen zu beteuern, - dies alles in einem Szenario, auf dem kulturpolitische Katastrophenstimmung und freiheitsbeflügelte Zukunftseuphorie aufs wirkungsvollste kontrastieren. Inzwischen ist jedoch insgesamt auch eine gewisse Ermüdung in diesem Streit zu konstatieren, hier und da auch verbunden mit vorsichtigen Signalen zur Kompromißbereitschaft. 
In Ansehung dieser Diskussion soll gewiß nicht verkannt werden, daß beide Seiten Wahres und Bedenkenswertes, auch Kritisches, Besorgniserregendes, ja Warnendes aus unterschiedlichem Blickwinkel zur allgemeinen Beurteilung ausgebreitet haben. Freilich muß aber auch festgehalten werden, daß dabei immer wieder, oft in geradezu peinlicher Weise, erkennbar geworden ist, wieviele der Streiter, gerade auch aus den politischen Parteien, Rundfunkpolitik nicht anders denn als Machtpolitik zu begreifen vermögen, so daß ihre Argumente, auch wenn sie sich vordergründig noch so gemeinwohlbesorgt ausnehmen, letztlich nicht die bestmögliche Weiterentwicklung der Sache Rundfunk zum Wohle der deutschen Rundfunkteilnehmer im Auge haben, sondern Machterhalt und Machterwerb, die Eroberung oder Verteidigung partei-oder gruppen-politischer »Erbhöfe in den Rundfunkanstalten und damit die dauerhafte Inbesitznahme eines der wichtigsten Mittel zur Bildung und Beeinflussung öffentlicher Meinung in diesem Lande.

In diese Entwicklung ist die katholische Kirche, wie alle gesellschaftlich relevanten Gruppen, vor allem die sogenannten »Groß-Gruppen «, unmittelbar und auf vielfältige Weise einbezogen; sie ist in ihrem kirchlichen Selbstverständnis berührt. So steht sie vor der Notwendigkeit, ihren Standort im System des deutschen Rundfunks neu zu überdenken, - eine Aufgabe, die, soweit erkennbar, in den letzten Jahren entweder gar nicht oder nur zögerlich, sowie in einer Weise erfolgt ist, die wohl in erster Linie von dem mehr oder weniger offenen Bestreben gekennzeichnet war, nicht vorschnell nach Veränderungen des Status-quo im deutschen Rundfunk zu rufen. Nachdem jedoch nun der Dualismus von öffentlich-rechtlichem und privat-rechtlichem Rundfunk in den Mediengesetzen der deutschen Länder, wenn auch im einzelnen auf recht unterschiedliche Weise, festgeschrieben wurde, ist eine generelleBestandsaufnahme sowie eine Neubewertung der rundfunkpolitischen Position der katholischen Kirche zu beiden Arten von Rundfunk nicht nur möglich, sondern weitmehr noch angezeigt, ja dringend erforderlich. Sie sollte sich nicht nur auf das konzentrieren, was aus den Länder-Mediengesetzen unmittelbar an aktuellem Entscheidungsbedarf auf sie zukommt; dies wäre ein allzu kurzatmiges Verfahren. Vielmehr sollte die Überprüfung das ganze Feld künftiger kirchlicher Rundfunkarbeit umgreifen und klären, was dort von ihr unter nunmehr beträchtlich veränderten Rahmenbedingungen erwartet wird und geleistet werden kann, aber auch, was die Kirche selbst vom Rundfunk in seinen neuen, unterschiedlichen Erscheinungsformen für ihren Weltauftrag erwarten kann. Diese Standortführung erleichtert ein zusammengefaßter Rückblick auf die wichtigsten Stationen der deutschen Rundfunkentwicklung seit 1945, weil sich in ihr die aktuellen Probleme ausgeformt haben.

\section{IV.}

Die nach dem Ende des 2. Weltkrieges in den deutschen Ländern erlassenen Rundfunkgesetze, die bis zum Ende der 70er Jahre die alleinige Grundlage für die Veranstaltung von Rundfunk bildeten, hatten diesen ausschließlich in öffentlich-rechtlicher Organisationsform konstituiert. Dies war eine - damals gewisserweise natürliche Folge der Tatsache, daß Rundfunk in Deutschland seit seiner Entstehung in den 20er Jahren unbestritten als "öffentliche" Aufgabe angesehen worden war, und daß man für eine solche »öffentliche « Aufgabe die Organisationsform des öffentlichen Rechtes auch und gerade zu dem Zeitpunkt als genuin ansah, als der deutsche "Staats «Rundfunk der Weimarer Republik und des 3. Reiches den 2. Weltkrieg nicht überlebte, und der Gedanke, auch weiterhin Rundfunk in diesem Lande als staatliche Aufgabe zu betreiben, aus wohlerwogenen Gründen verworfen worden war. 
Dieser neue staatsfreie - oder zumindest staatsferne -, gleichwohl aber als öffentliche Aufgabe wahrgenommene und öffentlich-rechtlich verfaßte Rundfunk gründete auf der Vorstellung, alle nichtstaatlichen Gruppen, soweit sie für das gesellschaftliche Leben als »relevant« anzusehen waren, in die öffentliche Aufgabe Rundfunk dergestalt einzubinden, daß sie durch eigene Vertreter in den Beschlußorganen der Rundfunkanstalten deren Arbeit mitbeeinflussen, auf die Wahrung ihrer Gruppeninteressen im Rahmen eines gesellschaftlichen Gesamtkonsenses dringen und damit an der Sache Rundfunk unmittelbar und gleichberechtigt teilhaben konnten. Daß diese Organisation damals auch durch den Mangel an Sendefrequenzen vorgezeichnet war, ist zwar richtig; dennoch war es das zentrale Anliegen der »Väter « dieser Rundfunkorganisation, die gesellschaftlichen Gruppen im Staate so zur Mitwirkung am Rundfunk zusammenzuführen, daß sie diese Aufgabe in »souveräner Gleichheit « und Gleichberechtigung als gemeinschaftliche Aufgabe wahrnahmen.

Diese Organisationsform des Rundfunks hatte vor rund einem Vierteljahrhundert in dem Streit zwischen Bund und Ländern über die Errichtung einer Rundfunkanstalt nach Bundesrecht ihre erste Bewährungsprobe zu bestehen und wurde durch die Entscheidung des Bundesverfassungsgerichtes vom 28.2.1961, das sog. 1. Fernsehurteil, bestätigt. Der Teil der Entscheidung, der sich mit den Kompetenzfragen zwischen Bund und Ländern in Sachen Rundfunk beschäftigt, kann hier bis auf die Feststellung außer Betracht bleiben, daß das Gericht die Zuständigkeit der Länder für Rundfunkangelegenheiten bestätigte. Im übrigen legte es aber wichtige rundfunkrechtliche "Eckwerte« für Organisation, Betrieb und Programmgestaltung der Rundfunkanstalten fest. Es bekräftigte, daß der Rundfunk in der Bundesrepublik eine »öffentliche Aufgabe « ist, ließ aber gleichzeitig dem staatlichen Gesetzgeber freie $\mathrm{Hand}$ bei der Entscheidung, ob er diese öffentliche Aufgabe in öffentlich-rechtlicher oder privatrechtlicher Rechtsform organisieren wollte. Es bekräftigte, und zwar mit ausdrücklichem Verweis auf den Frequenzmangel, die damalige Rundfunkorganisation, legte zugleich aber, gestützt auf Art. 5 GG und eine daraus in den folgenden Jahren sehr konsequent weiterentwickelte Rechtsprechung, entscheidenden Wert auf die Sicherung der Meinungsvielfalt in diesem binnenpluralen System. Binnenpluralismus wurde damit zum unverzichtbaren Strukturelement des öffentlich-rechtlichen Rundfunks, zum Element der Sicherung der Meinungsvielfalt.

Sosehr diese höchstrichterliche Klärung zu der hroffnung berechtigte, daß damit für geraume Zeit an der Front der deutschen Rundfunkpolitik Ruhe eintreten würde, ging die Entwicklung bald andere Wege. Das binnenplural-öffentlich-rechtliche Rundfunkmodell geriet überraschend schnell erneut in den Sog heftiger Kontroversen. In Ansehung der technischen Grundlage dieses Modells, der Frequenzknappheit, zeigte sich zu Ende der 60er Jahre deutlich, daß diese in etwa einem Jahrzehnt zu einem guten Teil werde überwunden werden können. Damit stieg zugleich die Chance, zu einem solchen Zeitpunkt mehr Rundfunkveranstalter zulassen zu können. Ausländische Beispiele - sowohl in anderen europäischen Ländern als auch in den USA - bestätigten diese Erwartung. Daneben aber - und dies sollte sich auf die Dauer als die für den öffentlich-rechtlichen Rundfunk folgenschwerste Veränderung erweisen machte die zu Ende der 60er Jahre beginnende heftige Polarisierung der politischen Meinungen in der Bundesrepublik auch vor dem Rundfunk nicht halt. Immer häufiger und zahlreicher traten neben die Zustimmung zum Programm der öffentlichrechtlichen Rundfunkanstalten auch Klagen über dessen politische Einseitigkeiten, bis hin zum offenen Vorwurf parteipolitischer Indoktrination und massiver Unterstützung bestimmter politischer Parteien. Über die Auslegung der rundfunkrecht- 
lichen Begriffe "Meinungsvielfalt« und »Ausgewogenheit« des Programms wurde häufig, erbittert und kontrovers debattiert. Begriffe wie "Rotfunk « und "Schwarzfunk " kamen als Etikettierungen einzelner Programme oder gar bestimmter Anstalten in Mode.

Auch innerhalb der Rundfunkanstalten, insbesondere ihrer Organe, hatte sich, zunächst mehr oder weniger von der Öffentlichkeit unbeachtet, eine parteipolitisch motivierte »Fraktions «-Bildung vollzogen. In diesen Organen bildeten sich nämlich nunmehr trotz der Tatsache, daß die weit überwiegende Mehrheit ihrer Mitglieder aus unterschiedlichen gesellschaftlichen Gruppen kam, Zusammenschlüsse, die sich in leicht durchschaubarer und eben deshalb halb komischer, halb ärgerlicher Camouflage ihrer eigentlichen Absichten und Ziele verharmlosend "Freundeskreise « nannten. Mit ihrer Hilfe übernahmen nun die politischen Parteien über ihre Vertreter in diesen Organen zielstrebig die Rollenführung bei der Meinungsbildung in diesen Gremien. In diese grobschlächtige Aufteilung wurden bald auch die Vertreter jener Großgruppen - zumindest rechnerisch - einbezogen, zu deren Selbstverständnis Überparteilichkeit, zumindest aber Parteienferne, gehört. Vertreter der Kirchen kamen dabei kurzerhand »auf das Ticket« des CDU-»Freundeskreises«, wie man dies im Rundfunkjargon nicht sehr taktvoll nannte, Vertreter der Gewerkschaften auf das $»$ SPD-Ticket«. Bald mußten sich auch die anderen Vertreter gesellschaftlich relvanter Gruppen unter das "kaudinische Joch « dieser Aufteilung begeben. So konnte es kaum verwundern, daß sich angesichts derartiger Methoden der Grundgedanke der binnenpluralen Rundfunkordnung, nach der die gesellschaftlich relevanten Gruppen - und zwar die Parteien eingeschlossen - in "souveräner Gleichheit " am Rundfunk teilhaben sollten, immer mehr auflöste, und daß bei diesem - rechtlich zumindest fragwürdigen, wenn nicht gar rechtswidrigen - Verfahren die Vertreter der gesellschaftlich relevanten Gruppen als die eigentlichen Mitgestalter des öffentlichen Rundfunks je länger, desto stärker Gefahr liefen, in den Organen der Rundfunkanstalteı zu "Hintersassen « der politischen Parteien zu werden.

Für die Kirche war diese Entwicklung in doppeltem Sinne fatal. Zum einen widersprach sie offenkundig ihrem Selbstverständnis und nahm keinerlei Rücksicht auf den besonderen kirchlichen Auftrag im Rundfunk und die daraus folgende Notwendigkeit, in den Organen der Rundfunkanstalten Entscheidungen für jedermann erkennbar eigenständig und nicht in Zuordnung zu politischen Parteien zu treffen. Zum anderen erwies sich aber auch, daß im Sog dieser Fraktionierung die Bereitschaft der jeweils anderen Gruppe deutlich abnahm, für spezifische Anliegen der Kirche im Rundfunk, vor allem im Programmbereich, Verständnis zu zeigen. So wurde es den Vertretern der Kirche oft schwer, sich bei der anderen Gruppe mit dem Tadel an Sendungen Gehör zu verschaffen oder gar Rügen durchzusetzen, wenn Programme gröblich gegen Glaubensgrundsätze verstießen oder diese profanisierten oder ironisierten. Als einem »Freundeskreis« zugerechnet, geriet der Vertreter der Kirche in einem solchen Gremium bei den Angehörigen des anderen "Freundeskreises « leicht zu einem quasipolitischen Gegner; zumindest aber wurden seine Anliegen primär unter dem Blickwinkel betrachtet, ob sie der Politik des anderen »Freundeskreises« nützten oder schadeten.

Davon abgesehen, mußten die Kirchen aber auch, bedingt durch die politische Polarisierung und die allgemeine Säkularisierung des öffentlichen Lebens, immer häufiger bei den Programmachern der Rundfunkanstalten einen Verlust an Sensibilität gegenüber Glaubensfragen verzeichnen. Manche Programmacher gerieten in der Zwischen- 
zeit zu antikirchlichen Eiferern oder benutzten den Anspruch der Anstalten, sich Minderheiten in besonderer Weise zuzuwenden und deren Meinungen zu artikulieren, zu einer oft verzerrten Darstellung kirchlicher Positionen oder einseitigen Darstellungen von Glaubensfragen, - dies alles nach der schlechten journalistischen $\mathrm{Ma}$ xime »bad news - good news « und in Erwartung, ein die Kirche betreffendes Problem als skandalträchtigen Konflikt wirksam »verkaufen « zu können. Daß in manchen Rundfunkanstalten mittlerweile die Redaktionen für Kirchenfragen beseitigt oder in neue Redaktionseinheiten überführt worden sind, in denen die Angelegenheiten der Kirchen nur mehr als ein »Unterfall« gesellschaftlicher Entwicklungen betrachtet werden, rundet dieses Bild ab. Als Fazit der Entwicklung bleibt insoweit nur die ernüchternde Feststellung, daß die öffentlich-rechtlichen Rundfunkanstalten zwar im großen und ganzen stark genug waren, gestützt auf ihre Rechtsordnung, staatliche Eingriffe abzuwehren, daß sie diese Freiheit von staatlicher Ingerenz aber damit haben bezahlen müssen, in die Botmäßigkeit der politischen Parteien zu geraten oder zumindest dieser Gefahr immer stärker ausgesetzt zu sein. Daß die Vertreter der anderen gesellschaftlichen Gruppen dieser Entwicklung nicht den notwendigen Widerstand entgegensetzten, oder sich gar in sie haben einbinden lassen, muß im Interesse des theoretisch so überzeugend konzipierten binnenpluralen offentlich-rechtlichen Rundfunkmodells nicht nur beklagt werden; es hat auch die Stellung der Kirche in diesem Rundfunksystem unverkennbar schwieriger gemacht.

\section{V.}

Je deutlicher die vorskizzierten Entwicklungen zutage treten, desto intensiver wurden die Bemühungen, dem öffentlich-rechtlichen Rundfunk andere Formen von Rundfunk zur Seite zu stellen, die sich in Organisation und Programm als Kontrast zu den öffentlich-rechtlichen Rundfunkanstalten darstellten. Die - wohl auch unter dem Druck solcher Forderungen - von den Regierungschefs der Länder beschlossenen "Kabelpilotprojekte «, die u.a. den Zweck verfolgten, die »Akzeptanz alternativer Programme durch die Rundfunkteilnehmer zu testen, erreichten nur in RheinlandPfalz (Ludwigshafen) und, wenn auch verspätet, in Berlin die Qualität unbestreitbarer Kontrastveranstaltungen zum öffentlich-rechtlichen Rundfunk. Bayern war bei seinem Münchener Kabelpilotprojekt durch Art. 111a seiner Verfassung gehalten, eine Organisationsstruktur vorzugeben, welche dem Verfassungsgebot entsprach, daß »Rundfunk nur in öffentlicher Verantwortung und öffentlich-rechtlicher Trägerschaft « betrieben werden darf, wodurch sich in Ansehung der Beteiligung privater Rundfunkveranstalter an diesem Kabelpilotprojekt spezielle Probleme ergaben. Nordrhein-Westfalen hatte bei seinem Kabelpilotprojekt von vornherein gesetzlich festgeschrieben, daß nur die öffentlich-rechtlichen Anstalten WDR und ZDF als Programmveranstalter zugelassen werden; Private erhielten lediglich die Möglichkeit, als Programmzulieferer tätig zu werden.

Die Kabelpilotprojekte befanden sich noch teils im Stadium ihrer Vorbereitung, teils hatten sie ihre Tätigkeit eben erst begonnen, als sich bereits abzeichnete, daß die rundfunkpolitische Entwicklung zumindest insoweit auch über sie hinweggehen würde, als sie auf der Bereitschaft der Regierungschefs der Länder fußte, vor Ablauf dieser Projekte keine definitiven Entscheidungen in Ansehung der Zulassung privater Rundfunkveranstalter zu treffen. Sicherlich sind die Kabelpilotprojekte, die mit z. T. beträchtlichem wissenschaftlichem Aufwand in Gestalt von Begleituntersuchungen und beträchtlichen Kosten durchgeführt werden, nicht völlig wertlos geworden; ihre ursprüngliche Bedeutung als für alle Länder bestimmte grundlegende rundfunk-techni- 
sche und rundfunk-politische Testverfahren haben sie jedoch weitgehend verloren. Nicht zuletzt hat sich auch die Erwartung mancher Regierungschefs nicht erfüllt, mit ihrer Hilfe eine rundfunkpolitische »Atempause « einzulegen oder die Einführung privaten Rundfunks zumindest um einige Jahre hinauszögern zu können. Unbeschadet der gegensätzlichen Entwicklungen im deutschen Rundfunkbereich in diesen Jahren - hier verstärkte Regionalisierungsbemühungen öffentlich-rechtlicher Anstalten, dort Zulassung privater Rundfunkanbieter in Niedersachsen, Baden-Württemberg und über das Kabelpilotprojekt in Rheinland-Pfalz - war es wiederum eine rundfunk-rechtliche Entscheidung, welche die Entwicklung am meisten beeinflußte. Das saarländische Rundfunkgesetz hatte als erstes der deutschen Rundfunkgesetze neben den Vorschriften für die öffentlich-rechtliche Rundfunkanstalt "Saarländischer Rundfunk « auch Vorschriften über die Errichtung privat-rechtlicher Rundfunkunternehmen im Saarland formuliert. Diese waren wiederholt Gegenstand gerichtlicher Überprüfung. Die wichtigste von ihnen war jedoch das Verfahren vor dem Bundesverfassungsgericht, das mit der Entscheidung vom 16.1.1981, dem sog. FRAG-Urteil, endete. Auch diese Entscheidung des Bundesverfassungsgerichtes enthielt, wie schon das 1. Fernsehurteil, nicht nur Ausführungen, welche allein das angegriffene Rundfunkgesetz betrafen. Vielmehr nahm das Bundesverfassungsgericht offenkundig Veranlassung, in Zusammenhang mit dieser Entscheidung seine bisherige rundfunkrechtliche Entscheidungspraxis im Angesicht der seit 1961 eingetretenen gewichtigen Veränderungen einer weitgehenden Prüfung zu unterziehen. Damit gewann die Entscheidung grundsätzliche Bedeutung, insbesondere für die in den folgenden Jahren erlassenen Mediengesetze der Länder. Dies rechtfertigt es, das FRAG-Urteil nachfolgend in seinen für die nachfolgende Mediengesetzgebung wichtigsten Positionen darzustellen.

\section{VI.}

Das FRAG-Urteil setzt die bisherige Linie der rundfunk-rechtlichen Rechtsprechung des Bundesverfassungsgerichtes insoweit konsequent fort, als auch in dieser Entscheidung der aus Art. 5 GG hergeleitete Grundsatz der »Meinungsvielfalt» im Zentrum der Ubberlegungen und Wertungen steht. Nur von diesem Begriff her ist die Entscheidung zu verstehen und einzuordnen.

Das Gericht fordert für den Rundfunk als »Medium und Faktor des verfassungsrechtlich geschützten Prozesses freier Meinungsbildung « »eine positive Ordnung, welche sicherstellt, daß die Vielfalt der bestehenden Meinungen im Rundfunk in möglichster Breite und Vollständigkeit Ausdruck findet, und daß auf diese Weise umfassende Information geboten wird «. Hierzu seien »Vorkehrungen zu treffen «, ”die sicherstellen, daß der Rundfunk nicht einer oder einzelnen gesellschaftlichen Gruppen ausgeliefert wird, daß die in Betracht kommenden gesellschaftlichen Kräfte im Gesamtprogramm zu Wort kommen, und daß die Freiheit der Berichterstattung unangetastet bleibt «.

Bei Zulassung privaten Rundfunks hält das Gericht es für ungewiß, ob im »Gesamtprogramm als Inbegriff aller inländischen Programme « "alle oder wenigstens ein nennenswerter Teil der gesellschaftlichen Gruppen und geistigen Richtungen auch tatsächlich zu Wort kommen, mithin ein Meinungsmarkt entsteht, auf dem die Vielzahl der Meinungsrichtungen unverkürzt zum Ausdruck gelangt«. Da im Gegensatz zur Presse »auf dem Gebiet des privaten Rundfunks zumindest vorerst von einem solchen Zustand nicht ausgegangen werden « könne, zudem „bei einem Medium von 
der Bedeutung des Rundfunks die Möglichkeit einer Konzentration von Meinungsmacht und die Gefahr des Mißbrauchs zum Zwecke einseitiger Einflußnahme auf die öffentliche Meinung in Rechnung gestellt werden* müsse, liege es in der Verantwortung des Gesetzgebers, $» \mathrm{da} ß$ ein Gesamtangebot entsteht, in dem die für die freiheitliche Meinungsbildung konstitutive Meinungsvielfalt zur Darstellung gelangt«. Zugleich müsse der Gefahr begegnet werden, „daß auf Verbreitung angelegte Meinungen von der öffentlichen Meinungsbildung ausgeschlossen werden und Meinungsträger, die sich im Besitz von Sendefrequenzen und Finanzmitteln befinden, an der öffentlichen Meinungsbildung vorherrschend mitwirken «; wiewohl dies »nicht mit letzter Gewißheit möglich« sei, müsse eine »hinreichende Wahrscheinlichkeit bestehen, daß sich in dem gesetzlich geordneten Rundfunksystem eine gleichgewichtige Vielfalt einstellt. *

In der Gestaltung der rechtlichen Regelungen für die Zulassung privaten Rundfunks attestiert das Gericht dem Gesetzgeber erneut große Freiheit und verweist darauf, daß ihm insbesondere das Grundgesetz keine bestimmte Rundfunkorganisation vorschreibt. Gleichwohl bedürfe die Entscheidung über die Einführung privaten Rundfunks einer gesetzlichen Regelung, bei der ein gewisser materieller Regelungsbedarf für den Gesetzgeber bestehe. Dazu gehörten *Zulassungsregelungen und ein dafür vorgesehenes rechtsstaatliches Verfahren «, die Normierung meiner begrenzten Staatsaufsicht zur Einhaltung der Gewährleistung der Rundfunkfreiheit«, Vorschriften, »die im Rahmen des zugrunde gelegten Ordnungsmodells sicherstellen, daß der Rundfunk nicht einer oder einigen gesellschaftlichen Gruppen ausgeliefert wird, und daß die in Betracht kommenden Kräfte zu Wort kommen können «. Für den "Inhalt des Gesamtprogramms « verlangt das Gericht endlich "verbindliche Leitgrundsätze, die ein Mindestmaß an inhaltlicher Ausgewogenheit, Sachlichkeit und gegenseitiger Achtung gewährleisten «, wobei allerdings im außenpluralen Modell dem einzelnen Veranstalter "keine Ausgewogenheit " obliege; er bleibe indes "zu sachgemäßer, umfassender und wahrheitsgemäßer Information und einem Mindestmaß an gegenseitiger Achtung verpflichtet «, sei »an die Schranken des Art. 5 Abs. 2 GG gebunden « und habe »für den Jugendschutz Sorge zu tragen «.

Eine kritische Befassung mit dieser Entscheidung kann im Rahmen dieser Ausführungen nicht erfolgen. Aber auch der mit rundfunkrechtlichen Fragen nicht vertraute Leser wird bei der Lektüre der in ihr formulierten »Eckwerte « und "Vorgaben « für die rundfunkrechtliche Gesetzgebung der Länder über privatrechtlich verfaßten Rundfunk bald den Eindruck gewinnen, daß manche Ausführungen des Gerichts wichtige Fragen offen gelassen, andere, gar neue produziert haben.

Ebenfalls soll hier nicht der Frage nachgegangen werden, ob das Gericht sich vielleicht zu sehr vom tradierten Bild des öffentlich-rechtlichen Rundfunks, insbes. seiner Organisations- und Programmstrukturen, hat leiten lassen, ob ihm der gerade in der Programmgestaltung so andersartige privatrechtlich verfaßte Rundfunk in anderen europäischen Ländern oder gar in den USA hinlänglich bekannt war, oder ob das Gericht seine Entscheidung überhaupt auf ausländische private Programmveranstalter bezogen wissen wollte, zumal es über den deutschen Rechtsraum hinausgehende Rechtsordnungen gar nicht auf ihre mögliche Anwendbarkeit in diesem Fall untersucht. Endlich führt auch die gelegentlich gestellte Frage nicht weiter, ob gewisse in der rundfunkpolitischen Diskussion der Bundesrepublik oft - und manchmal aus recht durchsichtigen Gründen - überzeichnete negative Erscheinungsformen einer 
gewissen Art von privat-rechtlich verfaßtem Rundfunk nicht hier und dort in der Argumentation des Gerichts allzu deutliche Spuren hinterlassen haben.

Nicht übersehen werden kann jedoch, daß auch diese Entscheidung den rundfunkpolitischen Streit nicht wesentlich zu verschärfen vermochte, daß die Kontrahenten dieses Streites vielmehr auch dieser Entscheidung im Wege gegensätzlicher Interpretation neue Argumente für oder gegen die Zulassung privatrechtlich verfaßten Rundfunks entnommen haben.

\section{VII}

Die Mediengesetze der Länder sind in dieser Sicht vor allem unter zwei Aspekten von Interesse; einmal in Ansehung des Scheiterns aller Versuche der Regierungschefs der Länder, zu einer Verständigung wenigstens über die wichtigsten materiall-rechtlichen Regelungen für die Zulassung privatrechtlich - verfaßten Rundfunks in den Ländern zu gelangen, zum anderen hinsichtlich der - gerade auch durch diese Dissense entscheidend mitgeprägten - konkreten Ausgestaltung dieser Gesetze und der daraus folgenden Auswirkungen auf die privat-rechtlich verfaßten Rundfunkveranstalter.

Dass alle bisherigen Versuche, zu einer staatsvertraglichen Vereinbarung der Länder über die Veranstaltung privatrechtlich verfaßten Rundfunks in der Bundesrepublik zu gelangen, an grundsätzlichen politischen Gegensätzen ebenso gescheitert sind wie an z. T. kaum begreiflichen nachrangigen Detailfragen, beweist nur den tiefen rundfunkpolitischen Riß, der die beiden Ländergruppen der sog. "A-Länder« (SPD-regiert) und »B-Länder « (CDU/CSU-regiert) trennt. Daher überrascht es auch nicht, daß die derzeitigen Erkundigungen zwischen ihnen über diejenigen Materien, die bei einer staatsvertraglichen Regelung überhaupt noch als konsensfähig angesehen werden können, sich thematisch immer mehr verdünnen und anscheinend $\mathrm{z}$. Zt. nur noch um die Verteilung von Satellitenkanälen kreisen. Dieser Befund stellt das seit Jahren betrüblichste Armutszeugnis dar, das die von den Ländern entscheidend mitzutragende bundesstaatliche Ordnung der Bundesrepublik sich selbst ausstellen muß. Gerade im Rundfunkbereich, wo es für die Länder vor allem um die Wahrung einer ihrer letzten substantiell bedeutungsvollen und entwicklungsfähigen Kompetenzen geht, hätte alle Veranlassung bestanden, nach früher oft praktizierten und bewährten Vorbildern über den eigenen Schatten zu springen und im Wege der Selbstbindung das Gut einer Einigung in einem zentralen Bereich eigener Kompetenz vor das landesegoistische Streben nach Durchsetzung bestimmter Postulate zu setzen. Dies wäre zudem auch das sicherste Mittel gewesen, diesen Regelungsbereich vor dem Zugriff des Bundes oder der Europäischen Gemeinschaft zu bewahren, zumindest jedoch durch eine in sich geschlossene und praktizierbare Rechtsordnung der Länder den Umfang solcher Eingriffe wenigstens einigermaßen zu begrenzen.

Daß diese Gefahren für die Erhaltung einer im Kern ungeschmälerten Rundfunkkompetenz der Länder nichts weniger als Phantasien sind, läßt sich leicht nachweisen. Zum einen zeigt die im Juni dieses Jahres von der Bundesregierung vorgelegte Entschließung über ein »Programm zur Verbesserung der Rahmenbedingungen für den privaten Rundfunk «, wie sehr der Bund, auch wenn er sich dabei primär auf fernmeldetechnische Aufgaben konzentriert, die Entwicklung des Rundfunks beeinflussen kann und will; zum anderen kann es nur betrüblich stimmen, daß weiterführende Konzeptionen für den Rundfunk nur von ihm, nicht aber von den Ländern entwickelt werden können. Was die europäische Gemeinschaft betrifft, so hat die Kommission 
mittlerweile den Vorschlag für eine "Richtlinie des Rates über die Koordinierung bestimmter Rechts- und Verwaltungsvorschriften der Mitgliedstaaten über die Ausübung der Rundfunktätigkeit« erarbeitet und zur Diskussion gestellt. Darüber hinaus läßt die Kommission schon seit einiger Zeit immer deutlicher erkennen, daß sie die Überwindung der unterschiedlichen rundfunkrechtlichen Rechtssysteme in der Gemeinschaft für eine ihrer wesentlichen Aufgaben hält. Daß dabei gelegentlich auch so larmoyante Töne wie die Forderung nach einer Wahrung der »europäischen Identität « mit anklingen, entbehrt zwar in Ansehung des Zustandes der politischen Integration Europas nicht einer gewissen Komik, zeigt andererseits jedoch, daß solche Forderungen recht publikumswirksam formuliert werden.

Ausländische private Rundfunkveranstalter, die ihre Programme europa- oder gar weltweit konzipieren und in verstärktem Umfang auf den deutschen Rundfunkmarkt streben, beobachten die Entwicklung des innerdeutschen Rundfunkrechtes nur noch mit allen Zeichen der Verständnislosigkeit. Daß die Länder der Bundesrepublik wegen dieses Zustandes nicht gerade als ideale Niederlassungs-, Produktions- und Veranstaltungstandorte für privaten Rundfunk angesehen werden, kann kaum verwundern. Gerade von diesen europäisch oder gar weltweit operierenden Rundfunkveranstaltern geht daher auch ein starker Druck auf die Europäische Gemeinschaft aus, durch eine europäische Rundfunkordnung die freie und gleichmäßige Entfaltung privaten Rundfunks in allen Mitgliedsstaaten der EG zu verwirklichen. Auch anläßlich solcher Bemühungen muß weiterhin die vielerorts in der Bundesrepublik erhobene Forderung dringlich wiederholt werden, daß die Länder sich endlich entschließen, zur unverzichtbaren rundfunkrechtlichen Gemeinsamkeit zurückzukehren und staatsvertragliche Regelungen für die Veranstaltung von privatem Rundfunk in der Bundesrepublik zustande zu bringen; dabei geht es natürlich nicht um formalrechtliche oder Organisationsvorschriften, etwa für die Länderanstalten für Kommunikation, die Zusammensetzung ihrer Gremien u.ä., sondern um das materiell-rechtliche Gerüst für die Veranstaltung privaten Rundfunks.

VIII.

Welche Schwierigkeiten dem Versuch entgegenstehen, in allgemeinverständlicher Weise vergleichbare Übersichten über die Regelungen der Länder-Mediengesetze zustande zu bringen, zeigt schon der Medien-Bericht 85 « der Bundesregierung, der soeben veröffentlicht wurde. Er hat - bei einer sehr allgemeinen Bewertung - drei verschiedene Formen von Länder-Mediengesetzen ermittelt:

1. Gesetze zur Fortentwicklung des bisherigen Rundfunkrechtes, die teilweise bereits geltendes Landesrundfunkrecht ändern oder ergänzen (Novelle zum WDRGesetz 1985), teilweise aber auch neues Landes-Rundfunkrecht schaffen und oder - zugleich auch Regelungen für privaten Rundfunk treffen (Niedersachsen, Schleswig-Holstein);

2. Mediengesetze, die neben - vornehmlich für den privaten Rundfunk bestimmten - Vorschriften auch andere Kommunikationsdienste gesetzgeberisch einbeziehen (Baden-Württemberg, Bayern, Hamburg, Nordrhein-Westfalen);

3. Kabelpilot-Gesetze (Berlin, Nordrhein-Westfalen).

In diese Aufstellung sind aber auch die Vorschaltgesetze zur vorläufigen Regelung der Ausstrahlung privater Rundfunkprogramme über Kabelanlagen mit einzubeziehen, wie sie in Bremen und Nordrhein-Westfalen bestehen; endlich ist in diesem Zusam- 
menhang auch der Entwurf eines Hessischen Gesetzes über die Weiterverbreitung von Satellitenprogrammen zu beachten, der sich aber nicht als vorläufige, sondern als abschließende gesetzliche Regelung versteht.

Ein Versuch, diese Gesetze insgesamt einer vergleichenden Bewertung zu unterziehen, muß sich, wie nach vorstehender Aufzählung leicht verständlich ist, auf einige Schwerpunkte konzentrieren, wenn er sich nicht in für den Nicht-Fachmann kaum noch nachvollziehbare Laby rinthe der Diskussion verlieren will. Diese Schwerpunkte sind nachfolgend auch unter dem Blickpunkt der Interessenlage der Katholischen Kirche ausgewählt worden. Dabei sind die geltenden Medien-Gesetze der Länder, der Entwurf des Hessischen Gesetzes über die Weiterverbreitung von Satellitenprogrammen in der Fassung der Drucksache 11/5010 des Hessischen Landtages und der Entwurf des Landes-Mediengesetzes Nordrhein-Westfalen in der Fassung vom 20.5.1986 zugrunde gelegt worden.

Folgende Schwerpunkte bieten sich für eine vergleichende Untersuchung an:

1. Meinungsvielfalt - Ausgewogenheit,

2. Programmgrundsätze,

3. lokale Programme,

4. Sondervorschriften für Programme, die über Kabelanlagen verbreitet werden,

5. spezielle Regelungen, welche besonders die Kirche betreffen,

6. Finanzierung des privaten Rundfunks.

Vorab ist darauf hinzuweisen, daß mehrere Medien-Gesetze einleitende Vorschriften enthalten, in denen Begriffsbestimmungen vorgenommen werden (Baden-Württemberg, Berlin, Hamburg, Nordrhein-Westfalen, Schleswig-Holstein), während andere Gesetze solche Begriffe ohne besondere Definition in Gesetzestext verwenden (Niedersachsen, Rheinland-Pfalz, Saarland). Im Zusammenhang dieser Ausführungen sollen von diesen Begriffsbestimmungen die beiden Begriffe "Vollprogramme" und "Spartenprogramme hervorgehoben werden, weil einige Gesetze daran bestimmte Rechte der Kirchen gegenüber privaten Programmveranstaltern knüpfen.

Unter »Vollprogramm « verstehen die Gesetze im wesentlichen Programme mit festen Anteilen an Informations-, Bildungs-, Beratungs- und Unterhaltungssendungen, wobei in einigen Gesetzen für diese Programme zusätzlich bestimmte tägliche Mindestsendezeiten gefordert werden. Als »Spartenprogramme« werden Rundfunkprogramme mit im wesentlichen gleichen Inhalten definiert.

Im Zentrum aller mediengesetzlichen Regelungen stehen, wie nach der FRAGEntscheidung des Bundesverfassungsgerichtes nicht anders zu erwarten, Vorschriften über die "Gewährleistung der Meinungsvielfalt«, in einigen Gesetzen auch mehr oder weniger synonym mit "Ausgewogenheit « bezeichnet. In z. T. sehr detaillierten und auch komplizierten Vorschriften legen die Gesetze fest, wie diese Forderung zu verwirklichen ist bzw. was zu geschehen hat, wenn Meinungsvielfalt nicht, noch nicht oder nicht mehr vorliegt.

Auffallend ist dabei zunächst, daß kein Gesetzgeber sich der Aufgabe unterzogen hat, den Begriff "Meinungsvielfalt« oder "Ausgewogenheit« inhaltlich zu definieren, obwohl dies nach der verfassungsgerichtlichen Vorgabe, der Gesetzgeber habe insoweit "gesetzliche Vorkehrungen « zu treffen, nicht eben ferngelegen hätte. Ob man zur Definition dieser Begriffe wenigstens in etwa unterstützend verwenden kann, was die 
Gesetzgeber zur inhaltlichen Beschreibung des Begriffs "Vollprogramm « ausgesagt haben, scheint zumindest fraglich. Ebenso wenig läßt sich in den Gesetzen eine Aussage darüber finden, inwieweit, vor allem auf welche Teile des Gesamtprogramms eines Rundfunkveranstalters, der Begriff "Meinungsvielfalt « zu beziehen ist, ob sich in Sonderheit das Meinungsvielfaltsgebot allein oder vorwiegend auf die informierenden Programmteile im engeren Sinne (Nachrichten, Magazine, Features u.ä. mit vorwiegend politischem Gehalt) oder auf alle Programmteile erstreckt. Diese Fragestellung hätte umso näher gelegen, als das Bundesverfassungsgericht schon im 1. Fernsehurteil die Auffassung niedergelegt hat, daß nicht nur die inhaltliche Gestaltung von Informationssendungen, sondern auch die Auswahl von Musikprogrammen rundfunkrechtlich ein meinungsbildender Vorgang sein kann.

Zuzugeben ist freilich, daß der Gesetzgeber mit dem Versuch, den Begriff »Meinungsvielfalt « inhaltlich verbindlich zu umschreiben, in beträchtliche Schwierigkeiten geraten wäre, insbesondere dann, wenn er es unternommen hätte, den in der Tat fragwürdigen Versuch zu machen, eine solche Definition etwa von den Programmstrukturen des öffentlich-rechtlichen Rundfunks her zu entwickeln. Dies hätte nur zu einer Uniformierung der Gesamtprogramme aller Rundfunkveranstalter geführt und damit dem Meinungsvielfaltsgebot in einer außenpluralen Rundfunkordnung diametral widersprochen.

Gleichwohl zeigen die - insbesondere bei der Ausführung der Vorschaltgesetze mit dem Begriff $»$ Meinungsvielfalt « in der Praxis gewonnenen Erfahrungen, daß sich die zur Kontrolle dieser Vorschrift berufenen Stellen schwer tun, diesen Begriff praktikabel zu machen; müssen sie dann doch selbst inhaltliche Bewertungen und Abgrenzungen vornehmen, welche alle Mediengesetzgeber vermieden haben. Dies bedeutet zugleich, daß sich auch in Zukunft die Anwendung dieser Begriffe nicht reibungslos vollziehen wird.

Haben die Mediengesetze der Länder die inhaltliche Umschreibung des Begriffs "Meinungsvielfalt « auch vermieden, so haben sie teilweise - und dann wieder in jeweils unterschiedlicher Form - den Begriff dadurch zu konkretisieren versucht, daß sie die Erfüllung dieses Gebots von der Existenz und Tätigkeit einer bestimmten Mindestzahl privater Rundfunkveranstalter abhängig gemacht oder die öffentlichrechtlichen Rundfunkveranstalter in diese Bewertung einbezogen haben. Berlin und das Saarland sehen das Gebot der Meinungsvielfalt als erfüllt an, wenn die Gesamtheit der Programme der privatrechtlichen und öffentlich-rechtlichen Rundfunkveranstalter diesem Gebot gerecht wird. Baden-Württemberg legt fest, daß Meinungsvielfalt erreicht ist, wenn neben den öffentlich-rechtlichen Programmen mindestens zwei regionale oder örtliche bzw. drei überregionale Programme ausgestrahlt werden. Fehlt es daran, so kann die Zulassung privater Rundfunkveranstalter gleichwohl unter gewissen Voraussetzungen erfolgen, so etwa, wenn der Veranstalter von den wesentlichen Meinungsrichtungen im Verbreitungsgebiet getragen wird, oder wenn der Veranstalter durch innerorganisatorische Maßnahmen, z.B. durch Bildung eines Programmbeirates, zur Meinungsvielfalt beiträgt; anderenfalls muß sich der Veranstalter vor der Zulassung verpflichten, in seinem Programm einen »offenen Kanal « zu betreiben oder in anderer Weise Beiträge zuzulassen, die zur Meinungsvielfalt beitragen.

Bayern statuiert eine Berlin und dem Saarland vergleichbare Regelung; sie unterscheidet jedoch nicht zwischen öffentlich-rechtlichen und privat-rechtlichen Programme, da die Verfassung nur die ersteren zuläßt. 
Bremen fordert für die in Kabelanlagen weiterverbreiteten Rundfunkprogramme, daß jedes deutschsprachige Programm - außer Spartenprogrammen - dem Meinungsvielfaltsgebot entsprechen muß.

Hamburg bestimmt, daß jedes von einem privaten Rundfunkveranstalter ausgestrahlte überregionale oder regionale Vollprogramm »im wesentlichen« die Vielfalt der Meinungen zum Ausdruck bringen muß.

Niedersachsen verlangt, daß die Programme der privaten Rundfunkveranstalter insgesamt - ohne diejenigen der öffentlich-rechtlichen Rundfunkveranstalter - die bedeutsamen politischen, weltanschaulichen und gesellschaftlichen Kräfte zu Wort kommen lassen müssen. Jedes zugelassene Programm muß »als einzelnes « diesen Anforderungen genügen, es sei denn, die Ausgewogenheit ist in Verbindung mit anderen Programmen gewährleistet.

Nordrhein-Westfalen legt in seinem vorläufigen Weiterverbreitungsgesetz für die über Kabelanlagen herangeführten Programme fest, daß deren Gesamtheit - neben den öffentlich-rechtlichen Rundfunkprogrammen - die Vielfalt der bestehenden Meinungen im Rundfunk »in möglichster Breite und Vollständigkeit zum Ausdruck bringen soll «. Der Entwurf des Landesmediengesetzes sieht demgegenüber vor, daß jedes private Vollprogramm oder die Gesamtheit aller Programme dem Meinungsvielfaltsgebot entsprechen muß.

Rheinland-Pfalz statuiert ein frequenzspezifisches Regelungskonzept, mit dem es durch Frequenzzuweisungen an bestimmte Programmarten Meinungsvielfalt bewirken will.

Schleswig-Holstein legt fest, daß die Programme in ihrer Gesamtheit sicherstellen müssen, daß die bedeutsamen politischen, weltanschaulichen und gesellschaftlichen Kräfte und Gruppen angemessen zu Wort kommen müssen, und daß die Gesamtheit der Programme nicht einseitig einer Partei oder Gruppe, einer Interessengemeinschaft, einem Bekenntnis oder einer Weltanschauung dienen darf. Ausgewogenheit gilt als erreicht, wenn neben den Programmen öffentlich-rechtlicher Programmveranstalter mindestens vier tägliche, im Geltungsbereich des Grundgesetzes veranstaltete Vollprogramme derselben Programmart und Verbreitungstechnik empfangbar sind. Auch in diesem Falle kann die Anstalt jedoch feststellen, daß Ausgewogenheit nicht oder noch nicht besteht. Auf das Erfordernis der Verbreitung in derselben Technik wird verzichtet, wenn mindestens die Hälfte der Rundfunkteilnehmer in SchleswigHolstein an eine Kabelanlage angeschlossen ist. Wenn Ausgewogenheit nach diesen Maßgaben nicht besteht, muß jedes einzelne Vollprogramm und jedes einzelne Spartenprogramm, soweit es an der politischen Meinungsbildung teilnimmt, ausgewogen sein.

Ebenso vielfältig wie die vorskizzierten Forderungen an Meinungsvielfalt und Ausgewogenheit sind auch die Vorkehrungen für den Fall, daß beide nicht oder noch nicht vorliegen. Für diese Fälle obliegen den Anstalten, die für den privaten Rundfunk in den Ländern zuständig sind, bestimmte Pflichten und Maßnahmen, wobei die Problematik der Gewährleistung von Meinungsvielfalt bei technischen Kapazitätsengpässen eine besondere Rolle spielt.

Im Vorfeld solcher Engpässe sehen mehrere Gesetze das Verbot von MehrfachErlaubnissen an den gleichen Anbieter für verschiedene Veranstaltungsformen vor 
(Baden-Württemberg, Berlin, Hamburg, Niedersachsen, Rheinland-Pfalz und Schleswig-Holstein), wobei in einigen dieser Gesetze solche Verbote ausdrücklich mit Hinweis auf eine mögliche Gefährdung der Meinungsvielfalt gerechtfertigt werden, in anderen aber auch mit spezifischen medien-, insbesondere pressepolitischen Aspekten (Hamburg).

Treten Kapazitätsengpässe auf, so legen einige Gesetze (Baden-Württemberg, Hamburg, Saarland, Nordrhein-Westfalen und Schleswig-Holstein) fest, daß die jeweilige Anstalt zunächst eine Einigung zwischen den Bewerbern zu versuchen hat; andere Gesetze (Niedersachsen, Rheinland-Pfalz) lassen die jeweilige Anstalt nach Maßgabe bestimmter Vorgaben direkt die erforderliche Entscheidung treffen. Ist ein Einigungsversuch vorgeschrieben, so kann er mit dem Ziel einer Aufteilung der verfügbaren Sendezeit zwischen den Bewerbern oder der Koordinierung ihrer Programme oder der vorrangigen Erlaubniserteilung für zunächst je ein Vollprogramm in Hörfunk und Fernsehen unternommen werden.

Kommt eine Einigung nicht zustande oder ist sie nicht vorgeschrieben, so hat die jeweilige Anstalt eine Entscheidung über die Reihenfolge der Erlaubniserteilung zu treffen. Unter den dabei zu beachtenden Kriterien steht die Erfüllung des Meinungsvielfaltsgebotes häufig obenan (Baden-Württemberg, Niedersachsen, NordrheinWestfalen, Rheinland-Pfalz, Schleswig-Holstein), und zwar wiederum allein oder kombiniert mit anderen Kriterien. Zu diesen gehören etwa die Begünstigung von Anbietergemeinschaften mit unterschiedlicher gesellschaftlicher Struktur (Hamburg), oder die Erlaubniserteilung an Anbieter mit einer plural verfaßten Organisation, etwa in der Form eines Programmbeirates. Andere Gesetze fordern die Bevorzugung von Anbietern mit einem Programm, das »professionellen Ansprüchen « genügt, die »Programmzeit火 am weitestgehenden in Anspruch nimmt (Niedersachsen), das »finanzierbar « ist (Baden-Württemberg), oder das in gewissem Umfang Informationen über die politische Entwicklung des jeweiligen Landes in das Programm aufzunehmen bereit ist (Saarland, Schleswig-Holstein).

Darüber hinaus bringen einige Gesetze in die Entscheidung indirekt auch andere Forderungen an die Anbieter ein, indem sie etwa dezidierte Programmauflagen statuieren (z. B. bezüglich der Anteile von Eigen- und Fremdproduktionen am Gesamtprogramm, der Überlassung von Sendezeiten an gemeinnützige Organisationen oder als spezielle Auflagen für die Gestaltung informationsintensiver Programmteile mit Bezug auf die Meinungsvielfalt (Hamburg, Nordrhein-Westfalen).

Darüber hinaus kennen alle Gesetze Rücknahme und - oder - Widerruf erteilter Erlaubnisse unter verschiedenen gesetzlich festgelegten formal- oder materiellrechtlichen Voraussetzungen. Zu diesen Voraussetzungen gehört in den meisten Fällen auch das Recht des Erlaubniswiderrufs wegen schwerwiegender und - oder - mehrfacher, zuvor von der Anstalt formell beanstandeter Verstöße gegen das Gebot der Meinungsvielfalt.

Endlich sind in diesem Zusammenhang noch weitere Tatbestände von Bedeutung. Mehrere Gesetze schreiben vor, daß Anbieter mit ihrem Erlaubnisantrag ein Programmschema vorzulegen haben, aus dem die für die Erlaubnis zuständige Anstalt ersehen kann, wie die Anbieter dem Meinungsvielfaltsgebot Rechnung zu tragen gedenken (Baden-Württemberg, Hamburg, Niedersachsen, Nordrhein-Westfalen, Schleswig-Holstein); dabei wird in Einzelfällen den Anbietern auch auferlegt, Änderungen 
dieses Programmschemas, soweit sie für die Meinungsvielfalt von Belang sein können, der Anstalt mitzuteilen (Nordrhein-Westfalen). Im Einzelfall schwierig zu beurteilen ist die Frage, inwieweit die Gesetze das Meinungsvielfaltsgebot auf ausländische Programmveranstalter ausdehnen wollen; ausdrückliche diesbezügliche Regelungen sind kaum erkennbar, bis auf diejenigen Fälle, in denen einzelne Gesetze von »deutschsprachigen « Sendungen sprechen und damit offensichtlich auch ausländische Rundfunkveranstalter einbeziehen, die solche Sendungen ausstrahlen.

Alle Mediengesetze enthalten - über den Regelungskomplex nMeinungsvielfalt/Ausgewogenheit « hinaus - mehr oder weniger umfangreiche Programmgrundsätze, die für die Einzelgestaltung der Programme privater Rundfunkveranstalter verbindlich vorgeschrieben werden. Sie umfassen insbesondere Vorschriften über die Achtung der Menschenwürde und der religiösen und weltanschaulichen Überzeugung anderer, über die Achtung von Ehe und Familie, die Verteidigung der demokratischen Freiheit, die Forderungen der sozialen Gerechtigkeit (Hamburg und Nordrhein-Westfalen) sowie die Förderung der Gleichberechtigung von Frau und Mann (NordrheinWestfalen). Ebenso findet sich in diesen Programmgrundsätzen das Gebot, sich nicht gegen die Völkerverständigung, die freiheitlich-demokratische Grundordnung der Bundesrepublik sowie das Wiedervereinigungsgebot des Grundgesetzes zu wenden. Endlich gehören zu diesen Grundsätzen auch das Verbot der Aufstachelung zum Rassenhaß, der Verherrlichung von Krieg und Gewalt, das Verbot pornographischer Sendungen - teilweise unter ausdrücklichem Bezug auf $\$ 184 \mathrm{StGB}$ - sowie das Gebot der Beachtung der Jugendschutzvorschriften.

Während fast alle diese Programmgrundsätze nicht mit konkretisierenden Angaben verbunden werden, enthalten die Gesetze konkrete Regelungen zumeist nur für den Bereich des Jugendschutzes. Die Ausstrahlung von Programmen mit jugendgefährdendem Inhalt wird auf die Zeit des späten Abends (nach 22 oder 23 Uhr) verwiesen, oder der Veranstalter hat dafür Sorge zu tragen, daß auf sonstige Weise, etwa durch Verschlüsselung oder Kodierung, Kinder bzw. Jugendliche solche Progrmame nicht sehen können. Rheinland-Pfalz hat in sein Gesetz noch konkretere Jugendschutzvorschriften aufgenommen und schreibt außerdem vor, daß die dortige Landesanstalt einen ständigen Ausschuß für Jugendschutz zu bilden hat; er kann unmittelbare Eingriffe gegen private Rundfunkveranstalter in Angelegenheiten des Jugendschutzes empfehlen. Unklar und z. Zt. aufgrund eines von einer öffentlich-rechtlichen Rundfunkanstalt anhängig gemachten Verwaltungsstreites rechtshängig ist die Frage, ob die nach dem Bundesgesetz über die Verbreitung jugendgefährdender Schriften gebildete Bundesprüfstelle im Bereich des Rundfunks tätig werden, ob sie insbesondere Fernsehprogramme mit nach ihrer Auffassung jugendgefährdendem Inhalt nach den Vorschriften dieses Gesetzes indizieren kann.

Für die Veranstaltung von lokalem Rundfunk enthalten nur die Gesetze in Bayern, Hamburg, Nordrhein-Westfalen und Rheinland-Pfalz ausdrückliche Sondervorschriften, während in den anderen Gesetzen, zumindest nach ihrem Wortlaut, offenbleibt, ob die dort niedergelegten allgemeinen Regelungen für die Veranstaltung privaten Rundfunks auch Rundfunkveranstaltungen im örtlichen Bereich betreffen.

Bayern führt lokalen Rundfunk - entsprechend seiner Verfassungslage - in der Form örtlicher Kabelgesellschaften ein. Kommunale Gebietskörperschaften, örtlich gemeinnützige Organisationen mit kultureller Zielrichtung, örtliche Anbieter von Rundfunksendungen und örtliche Zeitungs- und Zeitschriftenverlage müssen, unbe- 
schadet der Teilnahme anderer, die Möglichkeit einer angemessenen Beteiligung erhalten. Eine örtliche Kabelgesellschaft kann nur genehmigt werden, wenn die bedeutsamen politischen, weltanschaulichen und gesellschaftlichen Auffassungen in ihrem Gebiet angemessen zu Wort kommen können und kein Beteiligter einen bestimmenden Einfluß ausüben kann. Jedermann kann den örtlichen Kabelgesellschaften nach Maßgabe ergänzender Satzungsregelungen durch die Landeszentrale Rundfunkprogramme und Sendungen anbieten.

Hamburg läßt örtliche Programme zu, um ortsbezogenen Gruppen oder Gemeinschaften zu ermöglichen, kulturelle Aktivitäten zu fördern und zur Meinungsbildung über das örtliche Geschehen beizutragen. Zugelassen werden nur Anbietergemeinschaften der ortsbezogenen Interessen, die ausschließlich gemeinnützige $Z$ wecke verfolgen. Vorrangig zuzulassen sind solche Anbietergemeinschaften, die mehrere unterschiedliche Kräfte umfassen und den Mitwirkenden einen angemessenen Einfluß auf die Programmgestaltung und -verantwortung ermöglichen.

Nordrhein-Westfalen bestimmt im Entwurf seines Landesmediengesetzes, daß der lokale Rundfunk dem Gemeinwohl verpflichtet sein muß, das öffentliche Geschehen im Verbreitungsgebiet darstellen soll und aus unterschiedlichen Inhalten mit wesentlichen Anteilen an Information, Bildung, Beratung und Unterhaltung zu bestehen hat; auch darf er sich nicht ausschließlich einer bestimmten Zielgruppe zuwenden. Jeder Anbieter muß im Rahmen seiner täglichen Sendezeit bis zu $15 \%$ für Beiträge nicht erwerbswirtschaftlich orientierter Organisationen, insbesondere mit kultureller Zielsetzung, gegen Erstattung der Selbstkosten zur Verfügung stellen. Anbieter können neben natürlichen und juristischen Personen des Privatrechtes auch Kirchen, Religionsund Weltanschauungsgemeinschaften sowie die jüdischen Kultusgemeinden am Ort sein. Gemeinden, Gemeindeverbände sowie gemeindliche Unternehmen und Vereinigungen können sich nur in der Form der Anbietergemeinschaft, und auch nur mit höchstens einem Drittel der Kapital- und Stimmrechte, beteiligen. Tageszeitungen mit einer marktbeherrschenden Stellung im Verbreitungsgebiet dürfen ebenfalls als Einzelanbieter nicht zugelassen werden. Mitwirken dürfen sie in einer Anbietergemeinschaft, in der sie jedoch nicht die Mehrheit der Kapital- und Stimmrechtsanteile besitzen dürfen. Für jeden Anbieter ist ein lokaler Medienrat mit neun Mitgliedern zu bestellen; ihm werden beträchtliche Aufsichts- und Entscheidungskompetenzen zugewiesen.

Rheinland-Pfalz sieht im Rahmen seiner frequenzorientierten Regelung auch die Zulassung lokalen Rundfunks vor, und zwar ohne weitere spezifische Vorschriften.

Die in Schleswig-Holstein geltende Regelung, die lokalen Rundfunk nur in der Form von Fensterprogrammen mit einem Programmanteil von höchstens einem Drittel pro Tag zuläßt, muß als Sonderform gewertet werden.

Hinsichtlich der Programme, die über Kabelanlagen ausgestrablt werden, enthalten die Gesetze in Baden-Württemberg, Hamburg, Nordrhein-Westfalen, RheinlandPfalz, Saarland und Schleswig-Holstein spezifische Regelungen. Dazu gehören zunächst Vorschriften darüber, ob und inwieweit solche Programme zeitversetzt oder inhaltlich verändert ausgestrahlt werden dürfen; soweit bestimmte Grenzen überschritten werden, unterwerfen einige Gesetze diese Rundfunkveranstalter voll den für private Rundfunkveranstaltungen allgemein geltenden Vorschriften. Hinsichtlich des Meinungsvielfaltsgebotes, der Ausgewogenheit und der Beachtung der Programm- 
grundsätze räumen einige Gesetze den über Kabelanlagen verbreiteten privaten Programmen einige, meist allerdings nicht sehr gewichtige Vergünstigungen ein, indem sie etwa festlegen, daß sie bestimmte dieser Vorschriften nur »im wesentlichen « zu erfüllen haben oder von ihnen ganz entbunden sind.

Alle Gesetze enthalten jedoch Bestimmungen, nach denen die für private Rundfunkveranstalter geltenden Werbevorschriften auch für die über Kabelanlagen verbreiteten Programme verbindlich gemacht werden. Eine singuläre Regelung findet sich in Schleswig-Holstein; nach ihr kann die Genehmigung für ein über Kabelanlage verbreitetes Programm widerrufen werden, wenn es »nur auf geringes Interesse der Teilnehmer stößt«.

Die Mitwirkung der Kirche am privatrechtlich verfaßten Rundfunk ist in allen Ländergesetzen Gegenstand spezieller Regelungen, wenn diese auch in Einzelheiten deutlich differieren. Drei Bereiche sind besonders bedeutungsvoll.

Zum ersten räumen alle Gesetze - mit Ausnahme von Berlin - der Kirche Sitz und Stimme in dem zentralen Organ der jeweils für den privaten Rundfunk zuständigen Landesanstalt ein; es wird Medienrat, Medienbeirat, Anstaltsversammlung oder auch Vorstand genannt und besteht aus einer unterschiedlichen Zahl von Mitgliedern, die zwischen 5 (Berlin) und 49 (Bayern) schwankt, im Durchschnitt aber bei $28-30$ Mitgliedern liegt. In diesem Organ, das - bis auf das Gebot der Staatsfreiheit - nur äußerliche Ähnlichkeit mit dem Rundfunkrat eines öffentlich-rechtlichen Rundfunkveranstalters besitzt, nimmt die Kirche als eine von vielen gesellschaftlich relevanten Gruppen gleichberechtigt mit den anderen fast ausschließlich Aufsichts- und Kontrollfunktionen rechtlicher Art wahr; darüber hinausgehende Möglichkeiten einer Einflußnahme auf die Programme der privatrechtlichen Rundfunkveranstalter hat sie dabei nicht.

Zum zweiten kann die Kirche als Anbieter privatrechtlich organisierten Rundfunks in allen Ländern - mit Ausnahme von Bayern und Hamburg - uneingeschränkt zugelassen werden. Für Bayern ergibt sich auch insoweit die bereits dargestellte verfassungsrechtliche Sondersituation; Hamburg läßt Kirchen nur in Anbietergemeinschaften als Mitveranstalter zu.

In diesem Zusammenhang ist jedoch zu beachten, daß die Möglichkeiten der Kirche, als Veranstalter allein aufzutreten, faktisch durch das Meinungsvielfaltsgebot, die Ausgewogenheitspflichten in Ansehung des Programms sowie die Programmgrundsätze in ihren diversen Ausformungen in den einzelnen Gesetzen, vor allem aber durch die Regelungen für den Fall von Kompetenzengpässen, deutlich eingeschränkt sein können; dies muß vor allem dann gelten, wenn die Kirche sich aufgrund ihres Selbstverständnisses allein oder überwiegend an katholische Rundfunkteilnehmer wenden oder das Programm so gestalten will, daß katholische Glaubensüberzeugungen im Zentrum des Gesamtprogramms stehen.

Was endlich den lokalen Rundfunk betrifft, so kann die Kirche sich dort nur - mit Ausnahme von Nordrhein-Westfalen - in örtlichen Anbietergemeinschaften als Träger eines inhaltlich unterschiedlich strukturierten Programms oder als Zulieferer von Einzelprogrammen, nicht aber als eigener Rechtsträger von Rundfunk betätigen. $\mathrm{Da}$ neben wird sie in Nordrhein-Westfalen in lokalen Medienräten beteiligt sein. 
Zum dritten enthalten die gesetzlichen Regelungen auch Vorschriften über die Bereitstellung von Sendezeiten für Gottesdienste und kirchliche Handlungen sowie religiöse Sendungen; solche Vorschriften finden sich in Hamburg, Niedersachsen, Nordrhein-Westfalen, Rheinland-Pfalz, Saarland und Schleswig-Holstein.

Diese Vorschriften ähneln sich inhaltlich insoweit, als zumeist nur Vollprogrammen die Verpflichtung auferlegt wird, solche kirchlichen Sendungen auszustrahlen. Anspruch auf die Bereitstellung von Sendezeiten für solche Sendungen haben die $\mathrm{Kir}$ chen, die Religions- und Weltanschauungsgemeinschaften des öffentlichen Rechtes sowie die jüdischen Kultusgemeinden. Fast alle Gesetze legen den privaten Veranstaltern in diesem Zusammenhang die Pflicht zur Bereitstellung "angemessener Sendezeiten « auf; Rheinland-Pfalz limitiert diese Pflicht auf maximal " 90 Minuten pro Woche . Sendezeiten dieser Art sind im allgemeinen »auf Wunsch « oder »Verlangen « der Kirchen bereitzustellen. Endlich bestimmen die Gesetze, daß in solchen Fällen die privaten Rundfunkveranstalter für die Bereitstellung der Sendezeit "Erstattung der Selbstkosten « fordern können; die Regelung in Rheinland-Pfalz spricht von »angemessenen Kosten «.

Aus dem Komplex der gesetzlichen Regelungen über die Finanzierung des privaten Rundfunks ist hier zunächst darauf zu verweisen, daß alle Ländergesetze die privaten Rundfunkveranstalter von Einnahmen aus öffentlich-rechtlichen Gebühren ausschließen. Statt dessen haben sie sich aus dem "Finanzaufkommen des Veranstalters «, aus "Abonnements- und Einzelentgelten «, aus "Spenden " und aus "Werbung * zu finanzieren. Soweit bisher erkennbar, steht an der Spitze dieser Finanzierungsmöglichkeiten bei allen privaten Rundfunkveranstaltern z. Zt. der Bereich der Werbeeinnahmen, soweit die privaten Veranstalter nicht wegen mangelnder Werbeeinnahmen gezwungen sind, Erträge aus anderen Bereichen ihrer Tätigkeiten in den privaten Rundfunk einzuschießen. Bei der Werbung dominiert z. Zt. deren herkömmliche Form als Produkt- oder Dienstleistungswerbung. An Bedeutung könnten in absehbarer Zeit aber auch Einnahmen aus "Sponsor «-Sendungen gewinnen - eine Finanzierungsart, bei der eine Sendung insgesamt durch einen Geldgeber produziert und dem Veranstalter zur kostenlosen Ausstrahlung überlassen wird, oder bei der ein Geldgeber die Aufwendungen für die Herstellung einer Sendung des Veranstalters durch Geldzuwendungen oder andere wirtschaftliche Vorteile finanziert.

Im Rahmen dieser Darstellung kann auf eine finanzwirtschaftliche Bewertung dieses Finanzierungssystems, vor allem im Verhältnis zur Finanzierung des öffentlichen Rundfunks, verzichtet werden; Vorzüge und Nachteile einer reinen Gebühren- oder Werbeeinnahmen-Finanzierung liegen ohnehin auf der Hand. Hinzuweisen ist jedoch darauf, daß die Ländergesetze die Begriffe "Werbung " und "Sponsor-Sendung * teilweise unterschiedlich definieren, Umfang und Grenzen erlaubter Werbung in Details verschieden umschreiben, die sog. „Unterbrechungs-Werbung « - d.h. die Unterbrechung einer Sendung durch Werbeeinblendungen - rechtlich nicht einheitlich behandeln, und damit für den Bereich der Finanzierung des privaten Rundfunks teilweise nicht unerhebliche Unterschiede festschreiben.

Dies gilt auch für die Zulassung von Werbung an Sonn- und Feiertagen. Bisher ist diese gesetzlich nur in Baden-Württemberg für lokale und regionale Programme und im Entwurf des Gesetzes in Nordrhein-Westfalen untersagt; Bayern hat die dortige Landeszentrale gesetzlich ermächtigt, Werbung an Sonn- und Feiertagen durch Satzung einzuschränken. 
Diese auf sechs Schwerpunkte der Ländergesetzgebung begrenzte Analyse des gegenwärtigen Rechtes für private Rundfunkveranstaltung mag im Leser eine durchaus verständliche Verwirrung und Ratlosigkeit hervorrufen und ihn an Hamlet's Stoßseufzer erinnern: »s'ist ein wüster Garten «; insbesondere dann, wenn er sich vergegenwärtigt, daß diese Darstellung nur einen Teilbereich der Medien-Gesetze der Länder umgreift und auch deren übrige Bestimmungen in vielfältiger Weise, oft bis in kleine, bei erster Lektüre kaum feststellbare, gleichwohl aber wichtige Details, differieren. Angesichts dessen drängt sich die Frage auf, wie privat-rechtlicher Rundfunk sich in der Bundesrepublik überhaupt sachgerecht soll entfalten können.

Zugleich regt sich aber auch Zweifel, ob die Konkretisierung, welche die Ländergesetze dem vom Bundesverfassungsgericht statuierten Gebot der Meinungsvielfalt gegeben haben, richtig oder gar zwingend ist, nicht zuletzt angesichts der Tatsache, daß diese Konkretisierung zu einer gesetzlich verfestigten Rechtslage geführt hat, die dem privat-rechtlich verfaßten Rundfunk ganz außerordentliche, wenn nicht gar unverhältnismäßige Lasten auferlegt und seine Wettbewerbschancen im Verhältnis zum öffentlich-rechtlichen Rundfunk, jedenfalls fürs erste und in den entscheidenden Anfangsjahren, deutlich negativ verzerrt. So rechtlich unbestreitbar das aus Art. 5 GG deduzierte Meinungsvielfaltsgebot auch ist, so bleibt doch zu fragen, ob es in der Ausformung durch die Mediengesetze der Länder nicht zu einem "Fetisch" und damit "Wohltat« zur "Plage« geworden ist.

\section{IX.}

Versucht man aus alledem Folgerungen für eine zukünftige Rundfunkpolitik der katholischen Kirche zu ziehen, so muß am Beginn solcher Überlegungen gewiß die Einsicht stehen, daß es unerläßlich ist, von der grundlegenden Veränderungen im Rundfunk der Bundesrepublik nicht nur in einem formalen und reservierten Sinne Kenntnis zu nehmen, sondern sich mit voller Aufmerksamkeit auf diese Veränderung einzustellen; zugleich sollte auch der Meinung nicht weiter gefolgt werden, es handle sich bei dieser Entwicklung um ein zeitlich begrenztes Randphänomen, das sich infolge technischer, sachlicher oder finanzieller Schwierigkeiten in absehbarer Zeit von selbst erledigen werde. Die Tür zum privat-rechtlich verfaßten Rundfunk ist geöffnet; sie ist nicht mehr zu schließen.

Das aber bedeutet auch, daß es wenig sinnvoll ist, jene zeitweilig so beliebten Gedankengänge weiterzuspinnen, ob denn im Rundfunkbereich alles technisch Machbare auch gemacht werden sollte. Ganz gleich, ob man dies begrüßt oder bedauert: - die Entwicklung hat solche Erwägungen eindeutig überholt; und statt Verärgerung, Mißtrauen oder Resignation sollte nun eine von doktrinärer Enge ebenso wie von pragmatischer Unbekümmertheit entfernte Bereitschaft gefordert sein, »to make the best of it «.

Gewarnt werden muß auch davor, die Entwicklung des privaten Rundfunks vorwiegend am öffentlich-rechtlichen Rundfunk zu messen oder gar von diesem die alleinigen Maßstäbe der Bewertung zu nehmen. Abgesehen davon, daß der öffentlichrechtliche Rundfunk, auch bei aller Würdigung seiner Leistung, kein unbestreitbarer Maßstab für andere Formen des Rundfunks sein kann, muß dem privaten Rundfunk auch zugestanden werden, daß er unter der Prämisse angetreten ist, eine Alternative zum öffentlichen Rundfunk, gerade auch im Programmbereich, zu entwickeln. Gewiß gibt es da derzeit noch manches zu kritisieren; aber dabei sollte man nicht verken- 
nen, daß sich der private Rundfunk noch am Anfang seiner Entwicklung befindet, daß er sein Publikum noch suchen muß, und daß seine Programmstrukturen notwendigerweise mit seinen finanziellen Grundlagen und deren Entwicklung aufs engste verknüpft sind. Ungerechtertigt wäre es, diese Programmentwicklung von vornherein mit der Etikettierung »Kommerz-Funk « zu diskreditieren; ungerechtfertigt wäre es auch, sich bei solcher Betrachtung jenen anzuschließen, die in letzter Zeit mit auffälligem Eifer von der "kulturellen Aufgabe« oder gar »Mission « des öffentlich-rechtlichen Rundfunks reden. Offentlich-rechtlicher "Kultur-Rundfunk « gegen privatrechtlichen Kommerz-Rundfunk « zu setzen: - dies ist weder berechtigt noch zutreffend, sondern einseitig und unfreundlich. Sicher hat Rundfunk auch mit Kultur zu tun; aber Kultur hat auch mit Unterhaltung zu tun; deshalb ist der Anteil der Unterhaltungssendungen am Gesamtprogramm eines Rundfunkveranstalters kein Gradmesser für dessen Kultur oder Unkultur. Vor allem aber sollte endlich akzeptiert werden, daß nunmehr in der Rundfunklandschaft der Bundesrepublik ein "Markt « entsteht, auf dem Konkurrenten mit unterschiedlichen Angeboten um die Gunst des $\mathrm{Pu}$ blikums werben. Diesem Publikum als »Herrn « dieses Marktes sollte man daher die Möglichkeit des Mitreden-Könnens einräumen.

Was den öffentlich-rechtlichen Rundfunk betrifft, so zeigt sich, daß er auf die Veränderung der Verhältnisse zunächst zwiespältig reagiert. Einerseits sind Äußerungen der Selbstunterschätzung, verbunden mit der Forderung um Hilfe an den Staat, unüberhörbar; andererseits beginnt der öffentlich-rechtliche Rundfunk jedoch sehr bewußt, sich den Gesetzen des neuen Marktes anzupassen, indem er seine privaten Konkurrenten zumindest durch eine partielle Übernahme von deren Methoden - verstärkte Unterhaltungsanteile im Programm, Veränderungen der Programmpräsentation, verstärkte Bemühungen um den Werbemarkt - zu unterlaufen versucht.

Hier aber sind Zweifel angebracht, ob dies die richtige Reaktion ist. Ein insgesamt in technischer, personeller und finanzieller Hinsicht so gut fundiertes System sollte in dieser Lage davon absehen, in die Atritüde der Selbstunterschätzung zu verfallen. Statt dessen sollte der öffentlich-rechtliche Rundfunk ermutigt werden, sich bewußt auf seine Möglichkeit zu besinnen, anders zu sein als der private Rundfunk. Er sollte sich auf seine Verpflichtung aus der Binnenpluralität besinnen und deren Chancen besser nutzen. Mehr Ausgewogenheit der Programme bei gleichzeitiger Steigerung ihrer Aktualität, Verzicht auf parteipolitische Konfrontation, Abbau des »Bekenner-Journalismus «, nicht zuletzt auch der Verzicht auf jene "Monopolisten-Mentalität« im Verhältnis zum Rundfunkteilnehmer und bis hin zur Art und Weise der Verwendung seiner Finanzmittel, könnten dazu führen, daß der öffentlich-rechtliche Rundfunk, gestützt auf seine vielfältigen Ressourcen und Reserven, sein großes Erfahrungspotential und seine regionalen Kommunikationsmöglichkeiten, zu einer unverzichtbaren und eindrucksvollen Meinungsvielfalts-Reserve für den deutschen Rundfunk würde.

Die Vertreter der Kirche in den Organen der öffentlich-rechtlichen Rundfunkanstalten sollten solche Tendenzen bereitwillig unterstützen. Darüber hinaus dürfte es für die Kirche von Wichtigkeit sein, ob es gelingt, die neue Situation im Sinne der Wiederherstellung eines staats- und parteienfernen Binnenpluralismus zu nutzen, und zwar auch dann, wenn dies dazu führt, daß sich die Kirche mit der Rolle einer unter vielen gesellschaftlich relevanten Gruppen begnügen, sich in diesem Rollenverständnis bei Einzelfragen um ihr günstige Mehrheiten bemühen und zugleich nicht nur um die für eine Kirche wichtigen Anliegen, sondern um die Gesamtentwicklung des öffentlichrechtlichen Rundfunks kümmern muß. Umso mehr wird sie darauf bestehen 
können, auch im binnenpluralen System überall dort mitzureden, wo es nicht nur um kirchlich-religiöse, sondern auch um allgemein-ethische und humane Belange geht.

Im privat-rechtlich verfaßten Rundfunk sollte die Kirche die Chance dieses Systems für ihre eigene Mitwirkung unter Aufgabe ihrer bisherigen Zurückhaltung deutlicher ins Auge fassen. Sie sollte privaten Rundfunkveranstaltern ihre Mitarbeit anbieten und sich nicht nur auf die begrenzten Rechte beschränken, welche die MedienGesetze den Kirchen, etwa bei der Übertragung von Gottesdiensten, zugestehen. Auch privat-rechtlich verfaßte Rundfunkveranstalter, die in ihrem Programm ein breites Spektrum aller die Bevölkerung interessierenden Fragen in eingängiger Weise darbieten müssen, werden die Bereitschaft der Mitarbeit der Kirche würdigen. Zu fragen wäre auch, ob die Kirche nicht dazu beitragen kann, daß Programmangebote der privaten Veranstalter zustande kommen, in denen Anliegen der Kirche vermittelt werden, und zwar in allgemein verständlicher Sprache und zu Sendezeiten mit hoher Einschaltquote. So neu eine solche Überlegung auch sein mag, sie sollte nicht mehr von der Hand gewiesen werden. Dabei sollte man auch an das Institut der "SponsorSendung « denken, das die Möglichkeit bieten könnte, durch kirchliche Zuwendungen eine Programmproduktion zustande zu bringen, bei der allgemein interessierende Themen im Sinne katholischer Grundüberzeugungen vermittelt werden könnten. Freilich müßten für eine solche Programmproduktion wohl noch - wenn nicht alle, so doch die meisten - Voraussetzungen geschaffen werden. Gleichwohl ist nicht zu verkennen, daß ein solcher aktiver Einsatz bei der Programmgestaltung privater Rundfunkveranstalter eine der neuen Situation angemessene, ja notwendige Überlegung sein müßte.

In den Entscheidungsorganen der Medienanstalten für den privaten Rundfunk sollten die Vertreter der Kirche sicherlich alles tun, um allen gesellschaftlich relevanten Gruppen gleiche Mitwirkungsrechte zu garantieren und damit die partei- oder gruppenpolitische Fraktionierung ver- oder behindern. Vor allem aber sollten sie dazu beitragen, unvernünftige Hemmnisse, die der Entfaltung privatrechtlichen Rundfunks entgegenstehen, durch eine sachbezogene, großzügige Handhabung der Gesetze abzubauen.

Wichtigste Überlegung in diesem Zusammenhang bleibt jedoch die Frage, ob - und falls ja, wie - die Kirche sich als privater Rundfunkveranstalter mit einem eigenen Programm in die privatrechtlich verfaßte Rundfunkordnung einfügen soll, das unverwechselbar die Stimme des katholischen Deutschland im Gesamtkonzert des deutschen Rundfunks zum Ausdruck bringt. Schwergewicht und Dringlichkeit dieser Fragestellung ist offenkundig; ebenso offenkundig ist jedoch leider, daß, soweit erkennbar, diese Frage bisher kaum in der ihr gebührenden Breite und Intensität oder gar Offenheit erörtert worden, sondern meist allzu schnell in Ansehung der bei einer solchen Entscheidung erforderlichen Finanzmittel mehr oder weniger deutlich ins Reich der Illusionen verwiesen worden ist. So unbestreitbar die Kostenproblematik zum Zentrum dieser Überlegungen gehört, - allein sollte sie dennoch die Diskussion nicht bestimmen. Dies hieße nämlich nicht nur, die nach wie vor ungebrochene Anziehungskraft des Rundfunks, insbesondere des Fernsehens, auf die Menschen unseres visuellen Zeitalters zu verkennen; es würde auch einen möglicherweise vorschnellen Verzicht der Kirche auf die Nutzung eines Kommunikationsmittels bedeuten, dessen Wert für die Verbreitung, Vertiefung und Verteidigung von katholischen Glaubensüberzeugungen und Grundwerten, und damit als Zeugnis katholischer Weltzuwendung, in anderen Ländern längst erkannt ist. Dabei ist die Frage, ob die Kirche selbst 
oder eine mit ihr verbundene, ihr nahestehende oder von ihr geförderte Einrichtung Träger eines solchen privatrechtlich verfaßten katholischen Rundfunkprogramms sein kann oder sollte, bei aller unbestreitbaren Wichtigkeit auch nicht überzubewerten; gerade in diesem Zusammenhang könnte auch erkundet werden, ob nicht die Suche nach einem neuen unkonventionellen Organisationsmuster für ein solches katholisches Rundfunkprogramm nicht zugleich die Frage der Mittelbeschaffung für ein derartiges Vorhaben mit ins Auge fassen könnte.

So unbestreitbar es endlich auch ist, daß ein solches Vorhaben eine gewaltige Arbeitslast verlangt, sollten dennoch ideenreiche und in Dingen der modernen Kommunikationsmittel erfahrene und bewährte katholische Persönlichkeiten dadurch eher herausgefordert als abgeschreckt werden. In jedem Falle aber erscheint es dringend geboten, diese fundamentale Fragestellung nicht mit vorschnellen, dazu etwa noch resignierenden $Z$ wischenbilanzen aus der Diskussion zu nehmen.

Unbestreitbar kann eine solche Diskussion auch nicht sachverständig geführt werden, wenn sie nicht zugleich eine nüchterne Analyse aller Möglichkeiten mit umgreift, welche der Kirche in dem sich nun entwickelnden dualen Rundfunksystem zur Verfügung stehen. Vor allem gilt es, sorgfältig zu sondieren bei den Aufsichts- und Kontrollrechten, an denen die Kirche als gesellschaftlich relevante Gruppe in beiden Rundfunksystemen teilhat und bei der Umfang und Grenzen ihrer Einwirkung auf den Rundfunk präzise und illusionslos zu ermitteln sind; zum anderen sind die eigenen materiellen Gestaltungsmöglichkeiten in gleicher Weise zu erkunden, welche sich der Kirche in beiden Rundfunksystemen, vor allem aber in dem sich nun entwickelnden außenpluralen Rundfunkmodell, eröffnen. Nicht zuletzt aber sind in diese Überprüfung auch alle anderen kirchlichen Kommunikationsmittel, insbesondere die Kirchenpresse und die kirchlichen Nachrichtendienste, einzubeziehen. Dann erst wird sich einigermaßen zuverlässig beantworten lassen, ob - falls ja, welche - Defizite für die Kirche im Bereich der modernen Kommunikationsmittel insgesamt bestehen, wie sie zu beseitigen sind, und ob das neue duale Rundfunksystem dabei dauerhaft helfen kann.

Über alledem aber muß und sollte die Erkenntnis stehen, daß der neue, außenplurale und weitgehend privatrechtlich verfaßte Rundfunk nur dann zu einer dauerhaften Entfaltung und Bewährung kommen kann, wenn man sein wichtigstes Wirkungsgesetz nicht außer acht läßt. Dieses Wirkungsgesetz ist das Gesetz der aktiven, eigenverantwortlichen und selbständigen Mitarbeit. Aufsicht, Kontrolle und Kritik dessen, was andere im Rundfunk tun, genügt hier nicht mehr; nur das Mithandeln kann bewirken, daß eigene Vorstellungen in diesem Bereich Verbreitung und Gehör finden. Diejenigen gesellschaftlichen relevanten Gruppen, die sich durch Nichtteilnahme von diesem außenpluralen Rundfunk selbst ausschließen, müssen nicht nur die Folgen tragen; sie werden dann, wenn sie dieses System und seine Erscheinungsformen kritisieren, nur auf begrenztes Gehör stoßen können. Sich nicht einsetzen heißt hier, sich ausschließen.

\section{Postskriptum}

Kurz vor Beginn der Drucklegung des vorliegenden Aufsatzes hat das Bundesverfassungsgericht am 4.11.1986 seine Entscheidung zum Niedersächsischen Rundfunkgesetz verkündet. In dieser Entscheidung hat das Gericht die im vorstehenden Aufsatz 
dargestellte Rechtsprechung zum »Meinungsvielfaltsgebot « zwar grundsätzlich und im wesentlichen unverändert bekräftigt, sie zugleich aber in 3 Punkten wie folgt akzentuiert:

1) In der dualen Rundfunkordnung der Bundesrepublik weist das Gericht den öffentlich-rechtlichen Rundfunkanstalten die Aufgabe der "Grundversorgung « der Bevölkerung mit Rundfunk zu, weil diese Anstalten über ihre »terrestrischen Programme nahezu die gesamte Bevölkerung erreichen und damit zu einem inhaltlich umfassenden Programmangebot in der Lage sind «; in der Wahrnehmung dieser Aufgabe finden zugleich »der öffentlich-rechtliche Rundfunk und seine besondere Eigenart ihre Rechtfertigung".

2) Solange dies geschieht, »erscheint es gerechtfertigt, an die Breite des Programmangebots und die Sicherung gleichgewichtiger Vielfalt im privaten Rundfunk nicht gleich hohe Anforderungen zu stellen wie im öffentlich-rechtlichen Rundfunk «. Gleichwohl müssen die vom Gesetzgeber zu treffenden »Vorkehrungen « für den privatrechtlichen Rundfunk »aber bestimmt und geeignet sein, ein möglichst hohes $\mathrm{Maß}$ an gleichgewichtiger Vielfalt im privaten Rundfunk zu erreichen«.

3) Grundsätzlich »genügt diesen » Anforderungen der Rundfunkfreiheit eine Konzeption der Ordnung privaten, durch Werbeeinnahmen finanzierten Rundfunks, welche neben derartigen 'Mindestanforderungen' zur Vielfalt und Ausgewogenheit« die Sorge für deren Einhaltung »einem externen, vom Staat unabhängigen ... Organ« überträgt.

$\mathrm{Ob}$ und inwieweit diese Entscheidung, die im übrigen auch von der Entwicklung zu einem europäischen, ja weltweiten Medienmarkt in der Bundesrepublik erstmals ausdrücklich Kenntnis nimmt, für die privatrechtlichen Rundfunkveranstalter gegenüber dem bestehenden Rechtszsutand fühlbare Erleichterungen bringen kann, ist heute noch schwer abzuschätzen; sicher scheint derzeit nur, daß dem Gesetzgeber bei der Gestaltung der Rechtsvorschriften für diesen Bereich etwas mehr Bewegungsspielraum gegeben wird, den er allerdings nach eigenem medienpolitischen Ermessen ausfüllen kann. 


\section{SUMMARY}

The author gives a survey on the changing legal conditions of broadcasting in Germany in consequence of the planned introduction of private ownership in broadcasting in the different federal States. Although private broadcasters are for the most part handicaped by the new legislation in comparison to their public competitors, the Catholic Church should use the possibilities offered in order to balance her deficits as far as modern communications is concerned.

\section{RESUME}

L'auteur donne une vue générale sur les changements des conditions légales en matière de radio/télévision en Allemagne, suite à l'introduction de la propriété privée des radios/télévisions dans les différents états fédéraux. Quoique les radios/téléviseurs privés soient pour la plupart handicapés par la nouvelle législation en comparaison de leurs adversaires publics, l'Eglise catholique devrait uriliser les possiblités qui s'ouvrent ainsi pour compenser son retard en matière de communication moderne.

\section{RESUMEN}

El autor presenta una visión general acerca de la nueva situación pólitica y jurídica en el ambiente de la radioteledifusión en Alemania Occidental, y como ella se presentará en los diferentes estados federales alemanes, cuando se introduzca la radiodifusión privada. A pesar de que con la nueva situación legal, las posibilidades de competencia de la radiodifusión privada resultan pública, la Iglesia católica debiera de aprovechar las posibilidades que están a disposición, para equilibrar su déficit en el plano de los medios de comunicación modernos. 